\title{
An Access Algorithm Capable of Predicting Contention Window in LAA Networks
}

\author{
Kuochih Chu*, TzuChi Huang and YuHong Deng \\ Department of Electronic Engineering, Lunghwa University of Science and Technology, Taiwan \\ ${ }^{*}$ Corresponding author
}

\begin{abstract}
In recent years, various novel services appear in Long Term Evolution (LTE) networks to attract many users but cannot satisfy requirements of users with the available network bandwidth. However, most countries have no licensed spectrum available to LTE networks. To resolve this problem and compete Wi-Fi, 3rd Generation Partnership Project (3GPP) proposes the Licensed Assisted Access (LAA) to make LTE networks work on unlicensed bands. In this paper, we do some research on LAA related mechanisms and propose a new algorithm for it. We use simulations to show that our proposal can give LTE networks lower average collision times and average delay time than LAA. Our algorithm is capable of improving LAA in the future.
\end{abstract}

Keywords-LAA; LTE; Wi-Fi

\section{INTRODUCTION}

LTE (short for Long Term Evolution) networks are the fourth generation of mobile phone mobile communication technology standard (4G) networks currently used by most users. While the number of the LTE network service subscribers gradually increases, the available network bandwidth of the LTE network is not enough for network operators to enable various applications with guaranteed quality of service (QoS). To resolve the shortage of network bandwidth, operators seek more spectrums to be used by the LTE network. However, most countries have no licensed spectrum available to LTE networks. Accordingly, operators need to allocate unlicensed bands to LTE networks instead.

Currently, well-known unlicensed bands include $2.4 \mathrm{GHz}$ and $5 \mathrm{GHz}$. However, $2.4 \mathrm{GHz}$ currently is occupied by standards of Wi-Fi, Zigbee and Bluetooth, which makes the band usually interfered with each other by the applications. In comparison to $2.4 \mathrm{GHz}, 5 \mathrm{GHz}$ has more network bandwidth and less interference, because it is currently only occupied by the standard of Wi-Fi. Accordingly, operators need to allocate $5 \mathrm{GHz}$ to LTE networks. To avoid the Wi-Fi applications suffering the interference from LTE networks, 3GPP proposes the LAA in Release $13[1,2]$. LAA regulates the requirement of using Clear Channel Assessment (CCA) before a device transmits data. If the channel is idle, data can be sent immediately; otherwise, the collision resolution algorithm should be executed to wait for the idleness of the channel if the channel is busy. LAA uses the Binary Exponential Backoff (BEB) algorithm to resolve the collision, and Wi-Fi uses the BEB algorithm too because of the simplicity in implementation [3]. However, the BEB algorithm basically does not have good performance and furthermore works worse when LTE and Wi-
Fi networks coexist. Accordingly, we propose a new access algorithm in this paper to give LTE and Wi-Fi networks better performance when they coexist.

The remaining parts of this paper are organized as follows. In Section II, we introduce the LAA working principle and discuss the related works. Section III introduces our proposal. Section IV is the simulation and analysis. We compare our proposal and other algorithms in different network scenarios in order to show that our proposal outperform other algorithms. Section $\mathrm{V}$ is the conclusion.

\section{LAA OVERVIEW}

LAA regulates some working principles to avoid the interference between Wi-Fi and LTE networks. According to the operation of the LAA [2]. A collision appears when more than two devices simultaneously transmit data. LAA uses the BEB algorithm to resolve the collision. The BEB algorithm defines $\mathrm{CW}_{\min }$ and $\mathrm{CW}_{\max }$ parameters. $\mathrm{CW}_{\min }$ defines the minimum value of the contention window while $\mathrm{CW}_{\max }$ defines the maximum value of the contention window. The device sets q identical to $\mathrm{CW}_{\min }$ when the device requires to transmit data for the first time. If the device meets a collision when transmitting data, it sets $\mathrm{q}$ to be the double of the last $\mathrm{q}$. The device repeats the procedure each time it meets a collision until $\mathrm{q}$ is set equal to $\mathrm{CW}_{\max }$. Since the BEB algorithm does not have good performance, many researchers have different proposals to improve its performance.

Song et al. [4] proposed the Exponential Increase Exponential Decrease (EIED) algorithm. Instead of setting the maximum contention window equal to $\mathrm{CWmax}$ like the $\mathrm{BEB}$ algorithm, the EIED algorithm uses the half of the contention window value last time the device successfully transmits data as the maximum contention window. Accordingly, the EIED algorithm can make the device much quickly and adjust its contention window value to the appropriate value when it meets a collision in a network having a high load. Chen et al. [5] used the State Keeping Exponential Backoff (SKEB) algorithm The SKEB algorithm makes the device having the small contention window in a network with a low load and the large contention window in a network with a high load. The SKEB algorithm changes the contention window value only when the device continues transmitting data successfully or unsuccessfully many times. Teng et al. [6] adopted the Two Phase Backoff Algorithm (TPBA) for the quick change of the load in a network. The TPBA defines a threshold value. When the contention window value of the device is less than the 
threshold value, the BEB algorithm is used. The contention window value is changed only when the device has the contention window value more than the threshold value and continues transmitting data successfully or unsuccessfully many times.

In summary of the existing methods, certain algorithms do not have better performance and certain algorithms work only for a specific load in a network. To deal with these issues, we propose a new access algorithm in this paper. The proposed access algorithm can predict the next random backoff number according to the current data transmission result of the device. Our algorithm can outperform other algorithms in a network having various loads.

\section{CONTENTION Window PREDICTION AlgorithM}

In this section, we introduce our proposed algorithm named the Contention Window Prediction Algorithm (CWPA). CWPA predicts the contention window value suitable to the data transmission of the device this time according to the history of data transmission. The major difference between CWPA and LAA is the way to choose the random backoff number. Next, we explain the method that CWPA chooses the random backoff number. We assume that base stations of Wi$\mathrm{Fi}$ and LTE are managed by the same administrator. Accordingly, we can get the random backoff number chosen by each device when the devices transmit data successfully. CWPA follows Eq. (1) to calculate the predicted gn suitable to the data transmission of the device this time.

$$
g_{n}=\left(\alpha g_{n-1}+(1-\alpha) g_{a v g}\right)
$$

where $\alpha$ is the parameter used to configure the degree that the predicted gn relies on the last random backoff number or the average random backoff number in the history. The administrator can configure the initial value of $\alpha$ according to the characteristics of the load in a network. If the change of the load in a network is small, $\alpha$ can be set smaller; otherwise, $\alpha$ can be set larger if the change of the load in a network is large. $\mathrm{g}_{\mathrm{n}-1}$ is the random backoff number chosen by the device last time it transmits data successfully. The device chooses a random backoff number ranging from 0 to $\mathrm{CW}_{\min }$ for the first time of data transmission. $\mathrm{g}_{\mathrm{avg}}$ is the average in the history, i.e. summing up all $\mathrm{q}$ chosen by the device it transmits data successfully and dividing the summation by the data transmission times.

After that, we follow Eq. (2) to configure $q$ as the upper bound of the random backoff number.

$$
\mathrm{q}=\mathrm{g}_{\mathrm{n}} * 2
$$

We multiply the predicted $g_{n}$ by 2 and use it to be the upper bound of the random backoff number, since we require the middle in the range of the random backoff numbers to be the predicted $g_{n}$. Next, the device chooses the random backoff number $\mathrm{N}$ ranging from 0 to $\mathrm{q}$.

Besides, $\alpha$ in CWPA will be adjusted dynamically according to the change of the load in a network. When the random backoff number used in a successful data transmission close to $g_{\text {avg }}$ lasts for two times, it implies that the load in a network stays in a stable status and selects the average of random backoff number from the history; we decrease $\alpha$ by 0.1 in order to make the predicted $g_{n}$ next time much close to $g_{a v g}$. Conversely, when the random backoff number used in a successful data transmission close to $\mathrm{g}_{\mathrm{n}-1}$ lasts for two times, it implies that the change of the load in a network is large and has a preference the last random backoff number; we increase $\alpha$ by 0.1 in order to make the predicted $g_{n}$ next time much close to $\mathrm{g}_{\mathrm{n}-1}$. We detail the flow chart of CWPA in Fig. I.

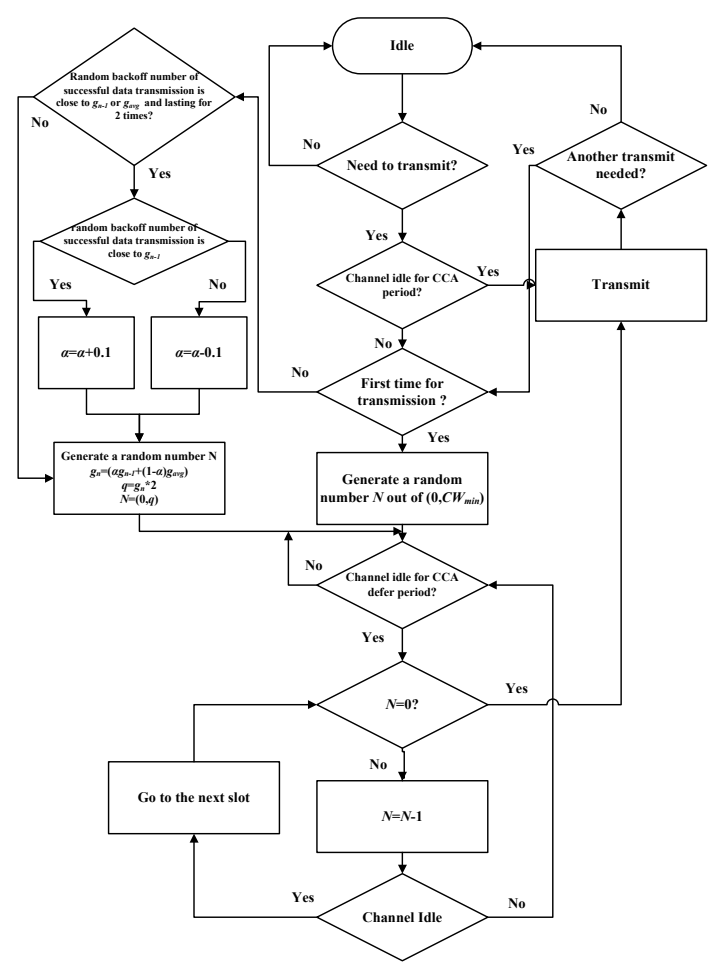

FIGURE I. CWPA FLOW CHART

\section{SimULATION AND ANALYSIS}

In this section, we introduce simulation parameters and compare simulation results. In simulation, we transmit 1000 records of data over the $100 \mathrm{Mbps}$ bandwidth network composed of nine Wi-Fi Access Points and one evolved Node B (eNB). In CWPA, we set 0.1 as value for $\alpha$ at the initiation. Tables I and II list the simulation parameters for four different scenarios briefed in Table III. Scenario A expresses that a network has the stable load all the time when devices transmit data, i.e. the load kept intact until the test end of the test. We simulate the network to have 10 different loads ranging from $10 \%$ to $100 \%$ and increase a load by $10 \%$. Scenario B expresses that the load in a network increases gradually and becomes $10 \%, 40 \%, 70 \%$ and $100 \%$, respectively, when 0,250 , 500 and 750 records of data are transmitted. Scenario C expresses that the load in a network changes quickly and becomes $50 \%, 100 \%, 50 \%$ and $100 \%$ for each time 250 records of data are transmitted. 
TABLE I. SCENARIO BRIEFS

\begin{tabular}{|l|l|}
\hline Scenarios & $\begin{array}{c}\text { Network Load for Each Time 250 Records of Data } \\
\text { Are Transmitted }\end{array}$ \\
\hline A & Fixed Load \\
\hline B & $10 \% \rightarrow 40 \% \rightarrow 70 \% \rightarrow 100 \%$ \\
\hline C & $50 \% \rightarrow 100 \% \rightarrow 50 \% \rightarrow 100 \%$ \\
\hline
\end{tabular}

TABLE II. LAA SIMULATION PARAMETERS

\begin{tabular}{|l|l|}
\hline \multicolumn{1}{|c|}{ Parameters } & Values \\
\hline Average Data Size (Bytes) & 368.1 \\
\hline Slot Size $(\mu \mathrm{s})$ & 9 \\
\hline CCA Time $(\mu \mathrm{s})$ & 34 \\
\hline Number of eNB & 1 \\
\hline Transmission Time of a Frame $(\mu \mathrm{s})$ & 30 \\
\hline Number of Transmission Data per eNB & 100 \\
\hline
\end{tabular}

TABLE III. WI-FI SIMULATION PARAMETERS

\begin{tabular}{|l|l|}
\hline \multicolumn{1}{|c|}{ Parameters } & \multicolumn{1}{c|}{ Values } \\
\hline Average Data Size (Bytes) & 368.1 \\
\hline$\left(\mathrm{CW}_{\min }, \mathrm{CW}_{\max }\right)$ & $(15,1023)$ \\
\hline Slot Size $(\mu \mathrm{s})$ & 9 \\
\hline DIFS Time $(\mu \mathrm{s})$ & 34 \\
\hline Transmission Time of a Frame $(\mu \mathrm{s})$ & 30 \\
\hline Number of Access Point & 9 \\
\hline $\begin{array}{l}\text { Number of Transmission Data per Access } \\
\text { Point }\end{array}$ & 100 \\
\hline
\end{tabular}

In simulation, we compare CWPA, LAA, EIED, SKEB and TPBA by observing their average collision times and average delay time. We use Eq. (3) to get the average collision times.

$$
\mathrm{C}_{\mathrm{avg}}=\mathrm{C}_{\text {total }} / \mathrm{N}_{\text {total }}
$$

where $\mathrm{C}_{\mathrm{avg}}$ is the average collision times, $\mathrm{C}_{\text {total }}$ is the total collision number, and $\mathrm{N}_{\text {total }}$ is the total data record number.

The higher the average collision times is, the more congested the network is. The device suffers more collisions before transmitting data successfully. If an algorithm can suppress the average collision times, it implies that the algorithm can make the device choose an appropriate random backoff number to have a short delay time. We use Eq. (4) to get the average delay time.

$$
\mathrm{T}_{\mathrm{avg}}=\mathrm{T}_{\text {total }} / \mathrm{N}_{\text {total }} \text {, }
$$

where $T_{\text {avg }}$ is the average delay time and $T_{\text {total }}$ is the total delay time. The higher the average delay time is, the longer the device has to wait before transmitting data successfully. The reason behind this is that the device suffers more collisions or chooses a larger random backoff number.

Fig. II shows the comparison of average collision times of different algorithms in Scenario A with configurations of different but stable loads lasting until the end of the simulation. Except for CWPA, other algorithms have similar average collision times. Because the load is fixed until the end of the test, the random backoff numbers chosen by the algorithms roughly are the same. Moreover, CWPA gets good performance too and has the lowest average collision times among the algorithms due to the selection of the random backoff number according to the history. Fig. III shows the comparison of average delay time of different algorithms in Scenario A with configurations of different but stable loads tested until the end of the simulation. Obviously, CWPA has the lowest average delay time among the algorithms and the difference of average delay time increases greatly according to the increase of the load configured in the network.

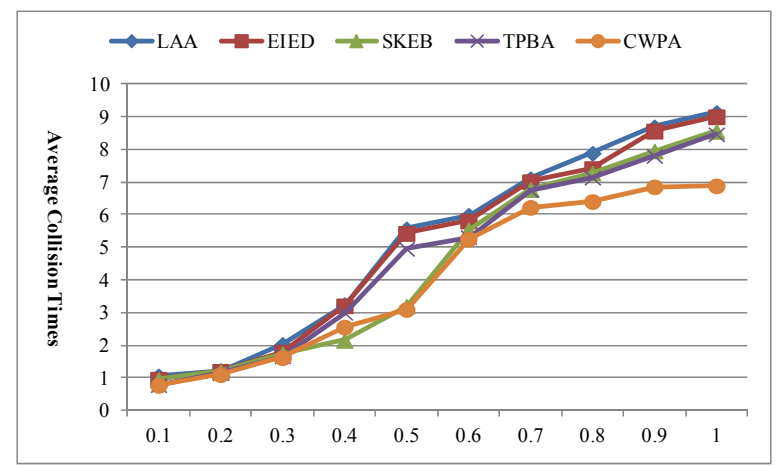

FIGURE II. COMPARISON OF AVERAGE COLLISION TIMES IN SCENARIO A

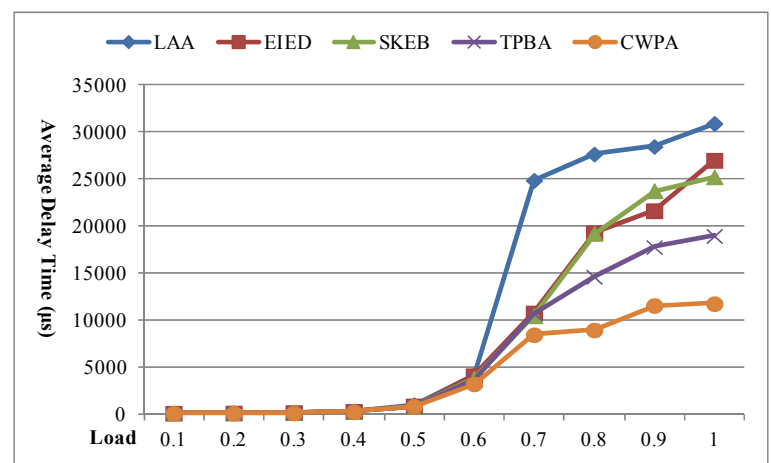

FIGURE III. COMPARISON OF AVERAGE DELAY TIME IN SCENARIO A

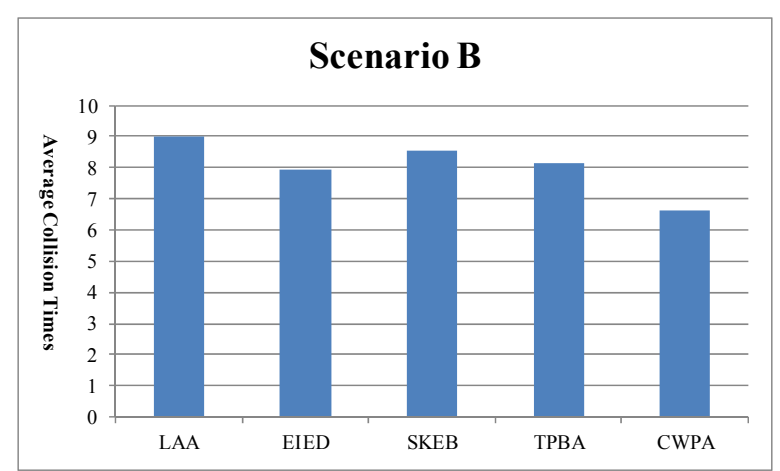

FIGURE IV. COMPARISON OF AVERAGE COLLISION TIMES IN SCENARIO B 


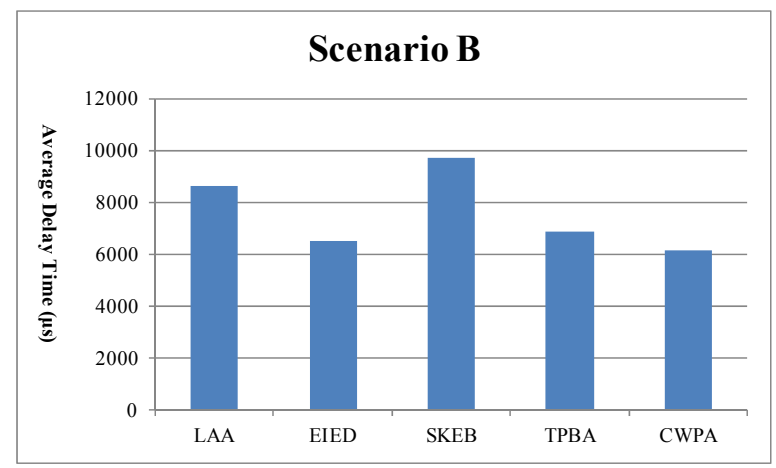

FIGURE V. COMPARISON OF AVERAGE DELAY TIME IN SCENARIO B

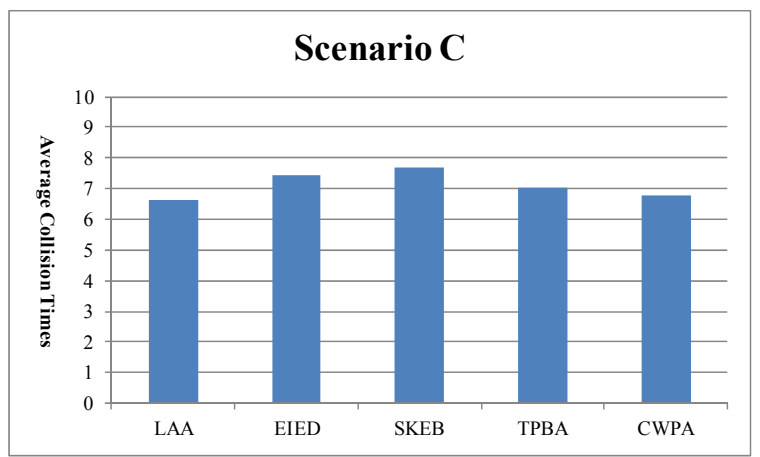

FIGURE VI. COMPARISON OF AVERAGE COLLISION TIMES IN SCENARIO C

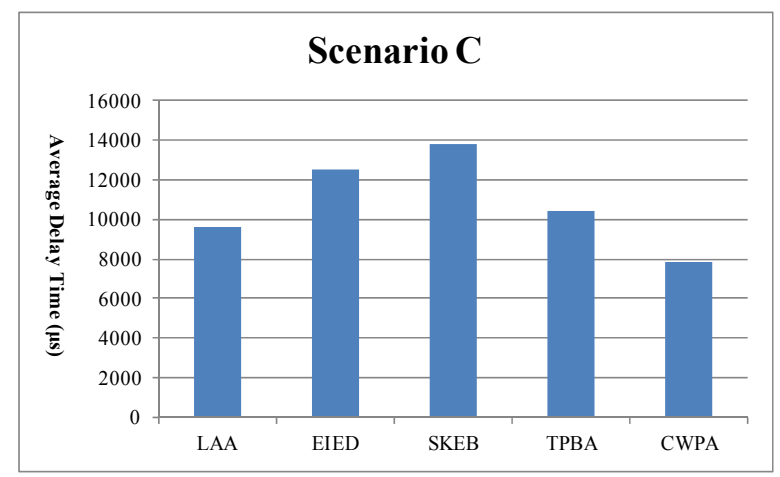

FIGURE VII. COMPARISON OF AVERAGE DELAY TIME IN SCENARIO C

Fig. IV illustrates the comparison of average collision times of different algorithms in Scenario B, where a network has different loads according to different numbers of data transmitted. Since CWPA adjusts $\alpha$ dynamically according to the increase of the load, it can predict the random backoff number much closer to the one suitable to the network condition. Accordingly, CWPA outperforms other algorithms in average collision times when the load in a network increases. Fig. V demonstrates the comparison of average delay time of different algorithms in Scenario B, where a network has different loads according to the number of data transmitted. Obviously, CWPA still has the lowest average delay time among the algorithms.
Fig. VI shows the comparison of average collision times of different algorithms in Scenario C, where a network has the load changed quickly. Since the load changes quickly in Scenario D, CWPA increases $\alpha$ gradually in order to make the predicted $\mathrm{g}_{\mathrm{n}}$ close to the last random backoff number. Therefore, CWPA can choose the random backoff number suitable to the network condition and have the lowest average collision times among the algorithms. Fig. VII shows the comparison of average delay time of different algorithms in Scenario C. CWPA has the lowest average delay time among the algorithms when the load in the network changes quickly.

According to simulation observations, CWPA outperforms other algorithms by having lower average collision times and average delay time in various scenarios where the network has different loads. Therefore, we consider that CWPA can be used to improve LAA to get better performance in LTE networks.

\section{CONCLUSION}

In recent years, LTE operators eagerly seek more spectrums in order to meet requirements of users. However, most countries currently have no licensed spectrum available to LTE networks. Accordingly, 3GPP requires to allocate unlicensed bands to LTE networks by proposing LAA to regulate the accesses to unlicensed bands. Since LAA does not have good performance, we propose a new algorithm named CWPA in this paper in order to have better performance. CWPA can predict the random backoff number used by a device for transmitting data next time according to the last random backoff number and the average of the random backoff numbers in the history. In simulation, we configure three scenarios for testing and comparing our CWPA to LAA, EIED, SKEB and TPBA. According to simulation observations, CWPA outperforms other algorithms by having lower average collision times and average delay time.

\section{REFERENCES}

[1] H. J. Kwon, J. Jeon, A. Bhorkar, Q. Ye, H. Harada, Y. Jiang, L. Liu, S. Nagata, B. L. Ng, T. Novlan, J. Oh, and W. Yi, "Licensed-Assisted Access to Unlicensed Spectrum in LTE Release 13," IEEE Communications Magazine, vol. 55, no. 2, pp. 201-207, Feb. 2017.

[2] S. Han, Y. C. Liang, Q. Chen and B. H. Soong, "Licensed-Assisted Access for LTE in Unlicensed Spectrum: A MAC Protocol Design," IEEE Journal on Selected Areas in Communications, vol. 34, no. 10 , pp. 2550-2561, Oct. 2016.

[3] Q. Zhang, Q. Wang, Z. Feng and T. Yang, "Design and Performance Analysis of a Fairness-Based License-Assisted Access and Resource Scheduling Scheme," IEEE Journal on Selected Areas in Communications, vol. 34, no. 11, pp. 2968-2980, Nov. 2016.

[4] N. O. Song, B. J. Kwak, J. Song, and L. E. Miller. "Enhancement of IEEE 802.11 Distributed Coordination Function with Exponential Increase Exponential Decrease Backoff Algorithm," in Proc. VTC 2003 (Spring), New Orleans, 2003.

[5] K. C. Chen, C. H. Lin, W. H. Huang and S. M. Chung, "State Keeping Exponential Backoff Mechanism for Wireless Channel Access Mechanism," in proc. 2012 Conference on Information Technology and Applications in Outlying Islands, Taiwan, 2012.

[6] J. C. Teng, W. H. Huang, Y. C. Wu, C. H. Lin and K. C. Chen, "A Two Stage Exponential Backward Algorithm for Improving Wireless Competitive Channel Mechanism," in proc. 2013 Conference on Information Technology and Applications in Outlying Islands, Taiwan, 2013. 